\title{
Co-occurrence of mutations in FOXP1 and PTCH1 in a girl with extreme megalencephaly, callosal dysgenesis and profound intellectual disability
}

\author{
Melinda Zombor ${ }^{1} \cdot$ Tibor Kalmár $\mathbb{B}^{1} \cdot$ Zoltán Maróti $^{1} \cdot$ Alíz Zimmermann $^{1} \cdot$ Adrienn Máté $^{1} \cdot$ Csaba Bereczki $^{1}$. \\ László Sztriha ${ }^{1}$
}

Received: 12 July 2018 / Revised: 3 August 2018 / Accepted: 17 August 2018

(c) The Author(s) under exclusive licence to The Japan Society of Human Genetics 2018

\begin{abstract}
Heterozygous disruptions in FOXP1 are responsible for developmental delay, intellectual disability and speech deficit. Heterozygous germline PTCHI disease-causing variants cause Gorlin syndrome. We describe a girl with extreme megalencephaly, developmental delay and severe intellectual disability. Dysmorphic features included prominent forehead, frontal hair upsweep, flat, wide nasal bridge, low-set, abnormally modelled ears and post-axial cutaneous appendages on the hands. Brain MRI showed partial agenesis of the corpus callosum and widely separated leaves of the septum pellucidum. Exome sequencing of a gene set representing a total of 4813 genes with known relationships to human diseases revealed an already known heterozygous de novo nonsense disease-causing variant in FOXP1 (c.1573C $>\mathrm{T}$, p.Arg525Ter) and a heterozygous novel de novo frameshift nonsense variant in PTCH1 (c.2834delGinsAGATGTTGTGGACCC, p. Arg945GlnfsTer22). The composite phenotype of the patient seems to be the result of two monogenic diseases, although more severe than described in conditions due to disease-causing variants in either gene.
\end{abstract}

\section{Introduction}

FOXP1 (forkhead box protein P1; Online Mendelian Inheritance in Man (OMIM) 605515) is one of the four members of the FOXP subfamily (FOXP1-4) and acts as a transcriptional repressor [1]. The FOXP1 gene is located on chromosome 3p13. Monogenic FOXP1 pathogenic variants and more extensive $3 p$ chromosomal deletions encompassing FOXP1 have been reported previously [2-4]. Eventually, a FOXP1 mutation-related phenotype (OMIM 613670) as a recognizable entity has been outlined with intellectual disability, specific language impairment with or without autistic spectrum disorder and dysmorphic features [3-5].

The PTCH1 (patched homologue 1, OMIM 601309) gene on chromosome 9q22.3 encodes a transmembrane

\footnotetext{
These authors contributed equally: Melinda Zombor, Tibor Kalmár

László Sztriha

sztriha.laszlo@med.u-szeged.hu

1 Department of Paediatrics, University of Szeged, Szeged, Hungary
}

glycoprotein [6] which functions as a hedgehog (HH) receptor and regulates $\mathrm{HH}$ signalling at the primary cilium $[6,7]$. Heterozygous germline $P T C H 1$ pathogenic variants cause Gorlin syndrome (OMIM 109400), an autosomal dominant disorder that predisposes affected individuals to developmental defects and tumourigenesis [6-8].

Megalencephaly is defined as an oversized brain with head circumference exceeding the age-related mean by two or more standard deviations [9]. A tendency to megalencephaly has been reported in both FOXP1- and PTCH1related conditions [3-5, 7-9]. We describe here a girl with co-occurrence of heterozygous disease-causing variants in both $F O X P 1$ and $P T C H 1$ genes in association with extreme megalencephaly, unreported in mutations of either gene, dysmorphic features, abnormal corpus callosum, and profound intellectual disability.

\section{Case report}

The proband, a girl, was born from the second pregnancy at 36 weeks of gestation via vaginal delivery with a birth weight of $2870 \mathrm{~g}(+0.4 \mathrm{SD})$, length of $50 \mathrm{~cm}(+0.8 \mathrm{SD})$ and head circumference of $37 \mathrm{~cm}(+1.8 \mathrm{SD})$. Dysmorphic features, 
such as prominent forehead, frontal hair upsweep, arched eyebrows, strabismus, broad nasal bridge, short nose with broad tip, long philtrum, pointed chin, low-set, abnormally modelled ears and symmetrical post-axial cutaneous appendages (skin tags) on both hands were observed (Fig. 1a, b, d). Progressive growth of her head circumference was noted (Fig. 1c); it was $58 \mathrm{~cm}(+6.1 \mathrm{SD})$ at the age of 4 years, and $61 \mathrm{~cm}(+7.1 \mathrm{SD})$ at the age of 8 years. Her height was $129 \mathrm{~cm}$ $(+0.2 \mathrm{SD})$ and weight $33 \mathrm{~kg}(+1.3 \mathrm{SD})$ at 8 years of age. Her motor and cognitive development was severely delayed. Brunet-Lézine test at the age of 2.5 years showed a developmental quotient of 40 . On examination, generalized hypotonia and preserved deep tendon reflexes were observed.

Brain magnetic resonance imaging (MRI) revealed partial agenesis of the corpus callosum (Fig. 1e) and widely separated leaves of the septum pellucidum (Fig. 1f). Brain computed tomography was not performed. Abdominal and cardiac ultrasound, chest X-ray and dental radiography were normal.

\section{Methods}

Routine chromosomal analysis was performed by standard techniques. Genomic DNA was extracted from peripheral blood samples with the Puregene kit (Gentra) after obtaining informed consent. Copy number changes were investigated by Affymetrix $750 \mathrm{~K}$ array. The Illumina Trusight One Exome Sequencing Panel (Illumina Inc., San Diego, CA, USA), covering the coding region of 4813 clinically relevant genes, was applied using Illumina MiSeq (Illumina Inc., San Diego, CA, USA). Variants were filtered based on severity and frequency against public variant databases including single-nucleotide polymorphism database (dbSNP), ClinVar, Exome Aggregation Consortium (ExAC), Exome Variant Server (EVS) and inhouse clinical exome database of 140 unrelated Hungarian persons.
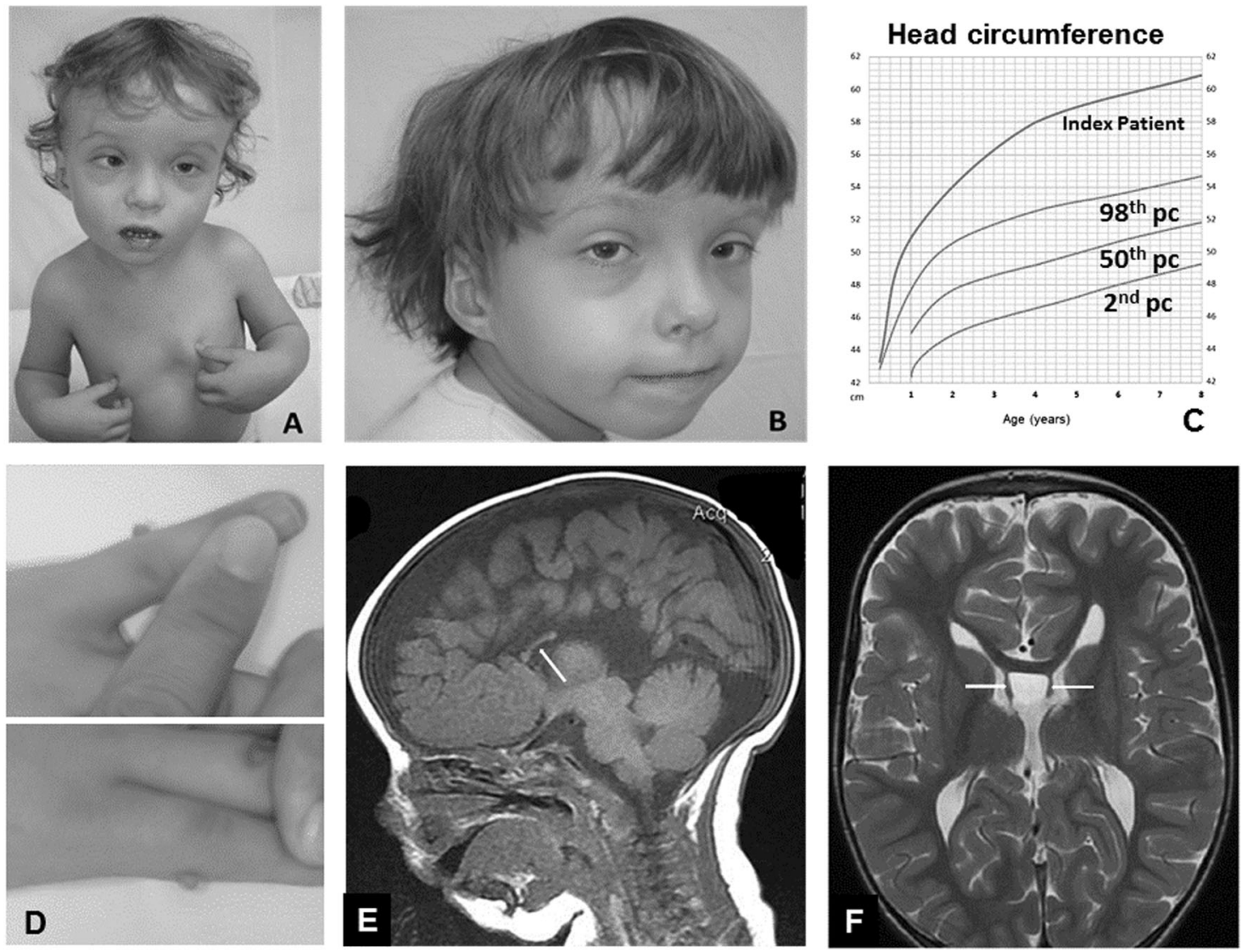

Fig. 1 Photographs, head circumference chart and brain MR images. a, b The patient at the age of 2 (a) and 6 (b) years. Note prominent forehead, arched eyebrows, strabismus, flat, wide nasal bridge, short nose with a broad tip, long philtrum, small lower jaw and low-set ears with abnormally modelled helices. $\mathbf{c}$ The chart shows the progressive growth of the head circumference. d Bilateral skin tags on the fifth fingers. e Sagittal T1-weighted MR image at the age of 3 months shows the partial agenesis of the corpus callosum with a thin remnant of the anterior part of the body (arrow). f Axial T2-weighted MR image at the age of 4 years demonstrates the thin anterior part of the corpus callosum, dilated ventricles and widely separated leaves (arrows) of the septum pellucidum 


\section{Results}

Chromosomal analysis showed a normal 46,XX karyotype. Copy number changes were not found by Affymetrix $750 \mathrm{~K}$ array. Clinical exome (Trusight One panel) sequencing revealed heterozygous variants in exon 18 of the FOXP1 (NM_032682.5:c.1573C >T, NP_116071.2:p.Arg525Ter) and exon 17 of the PTCHI (NM_000264.4:c.2834delGinsAGATGTTGTGGACCC, NP_000255.2:p.Arg945GlnfsTer22) genes in the patient (Figs. 2 and 3). The FOXPI variant has been reported earlier as a nonsense pathogen mutation (rs112795301, RCV000005214), while the PTCH1 variant (RCV000655932) has not been found in either dbSNP, ClinVar, ExAC or EVS databases or a cohort of 140 unrelated Hungarian controls. Sanger sequencing confirmed both variants as de novo mutations in the patient. They were not present either in the parents or in the patient's healthy brother (Figs. 2 and 3).

\section{Discussion}

We describe heterozygous disruptions in two genes, namely FOXP1 and PTCH1. Megalencephaly has been described in both FOXP1- and PTCH1-related conditions; however, such an extreme rate of head growth, as seen in our patient, has never been reported in association with disease-causing variants in either FOXP1 [3-5] or PTCH1 [8, 10-13] genes. Although structural brain abnormalities have been found occasionally in FOXP1-related disorders, dysmorphic corpus callosum has been reported only in a single case [3,4]. Brain malformations were also rarely reported in PTCH1 mutation [10-12], notwithstanding corpus callosum agenesis, dysgenesis and hypoplasia occurred in a few patients $[11,13,14]$. It seems that the intellectual disability and language deficit were less severe in children with mutations only in one of these genes [3-5, 8, 10-13]. We suggest that the effects of multiple hits, disruptive diseasecausing variants in two different genes simultaneously added and resulted in the serious composite clinical phenotype in our patient (Table 1).

Functional characterization of FOXP1 variants [4, 15] provided evidence that the de novo heterozygous nonsense mutation, c. $1573 \mathrm{C}>\mathrm{T}$, p.Arg525Ter, found in our patient, was pathogenic [4, 15]. FOXP proteins regulate gene expression by forming homo- and hetero-dimers with each other and they interact with other transcription factors forming a network involved in cortical development [16]. Studies on embryonic neural stem cells and mice with Foxpl down-regulation or deletion in the developing forebrain confirmed that an extensive signalling network regulated by Foxp1 is involved in important developmental processes such as neurogenesis, neuronal migration and differentiation [17-19].

Several hundred pathogenic variants in the PTCHI gene have been described [6,7]. The novel de novo heterozygous variant c.2834delGinsAGATGTTGTGGACCC, p. Arg945GlnfsTer22, in our patient causes truncation of one of the extracellular loops of the PTCH1 leading to the loss of a significant part of the C-terminal protein, including five transmembrane domains [6]. The PTCH1 protein is a member of the $\mathrm{HH}$ receptor complex in the primary cilia. It represses Smoothened (SMO), which is a member of the Gprotein-coupled receptor superfamily. The truncated PTCH1 protein probably fails to inhibit SMO activation resulting in dysregulation of the $\mathrm{HH}$ signalling pathway. We
Fig. 2 FOXP1 mutation. Mutation in FOXP1 gene (NM_032682.5) at c.1573 position results in a STOP codon. The mutation is very likely de novo since it was absent in the parents (a). The mutated position is highlighted in the DNA sequence of the affected child. Standard IUPAC (International Union of Pure and Applied Chemistry) nucleotide ambiguity DNA code $(\mathrm{Y}=\mathrm{T} / \mathrm{C})$ used (b)
A

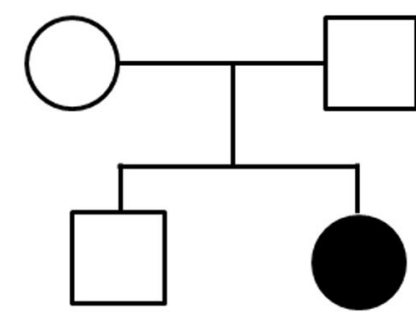

FOXP1 NM_032682.5:C.1573C>T NP_116071.2:p.Arg525Ter

De novo mutation

C522 F523 V524 R525 V526

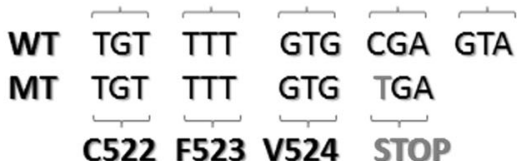

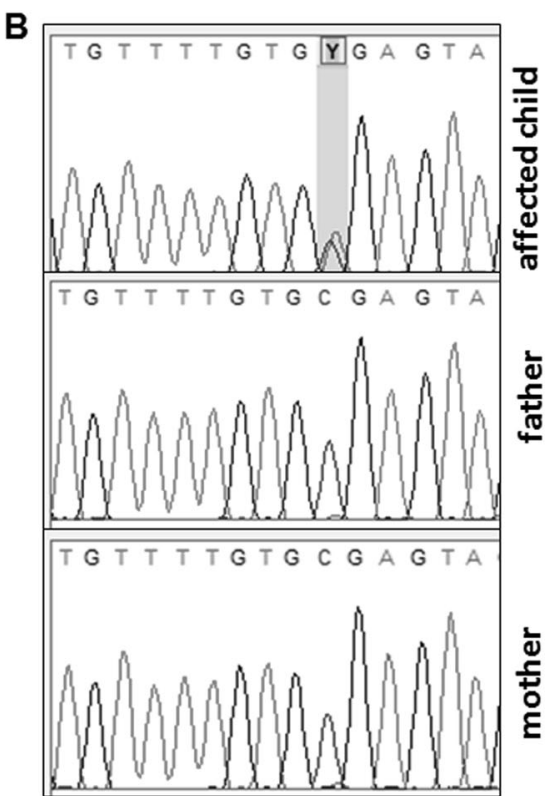


A

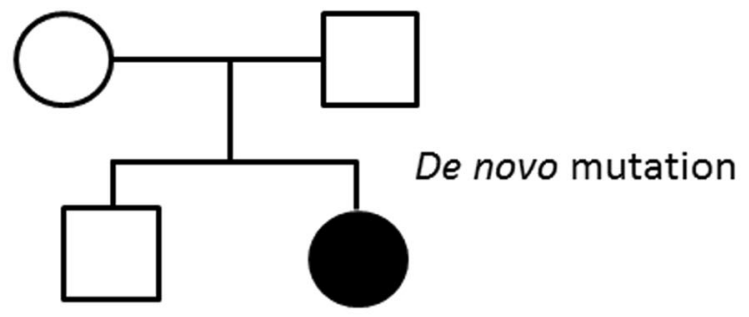

PTCH1 NM_000264.4:C.2834delGinsAGATGTTGTGGACCC NP_000255.2:p.Arg945GInfsTer22

V932 A933 Y934 A935 A936 S937 Q938 A939 N940 1941 R942 P943

WT GTC GCG TAT GCT GCC TCC CAG GCC AAC ATC CGG CCA

MT GTC GCG TAT GCT GCC TCC CAG GCC AAC ATC CGG CCA

V932 A933 Y934 A935 A936 S937 Q938 A939 N940 1941 R942 P943

B
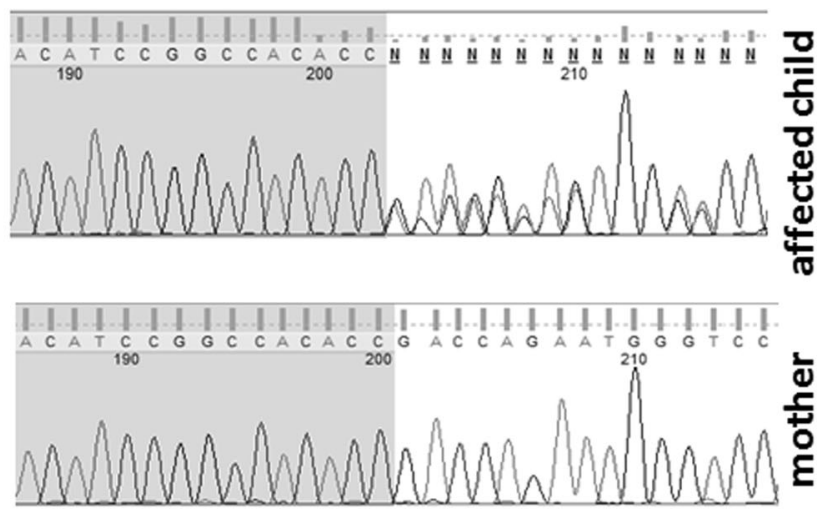

H944 R945 P946E947 W948 V949 H950 D951 K952 A953 D954 Y955

WT CAC CGA CCA GAA TGG GTC CAC GAC AAA GCC GAC TAC

MT CAC CAG ATG ITG TGG ACC CAC CAG AAT GGG TCC ACG

H944 Q945 M946 L947 W948 T949 H950 Q951 N952 G953 S954 T955

M956P957 E958T959 R960 L961 R962 1963 - intronic -

WT ATG CCT GAA ACA AGG CTG AGA A gtaagtagcgt

MT ACA AAG CCG ACT ACA TGC CTG AAA CAA GGC TGA

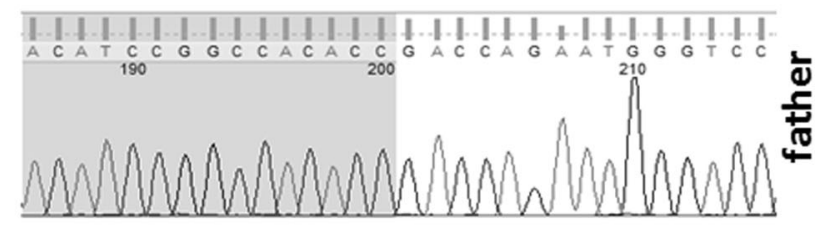

T956 K957 P958T959 T960 C961 L962 K963 Q964 G965 STOP

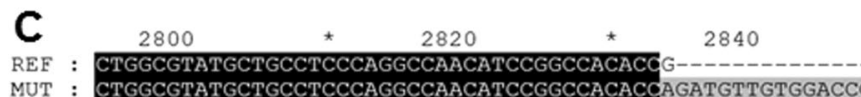

Fig. 3 PTCH1 mutation. a The complex PTCH1 variant caused by a single-nucleotide deletion and a $15 \mathrm{bp}$ long insertion in exon 17 which causes a premature STOP codon (NP_000255.2:p.Arg945GlnfsTer22).

The mutated portion of the sequence is highlighted in red. The mutation is very likely de novo since it was absent in the parents. b

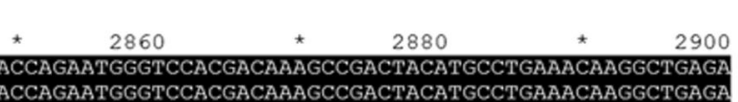

The presence of PTCH1 (NM_000264.4) mutation at DNA level confirmed by Sanger sequencing in the affected child. It was absent in the parents. The complex mutation starts after the highlighted portion of the sequence (blue). $\mathbf{c}$ The sequence alignment of the reference and mutated $\mathrm{PTCH} 1$ alleles
Table 1 Common versus distinct clinical features attributable to mutations in FOXP1 and PTCH1

\begin{tabular}{lll}
\hline & FOXP1 (refs. [2-5]) & PTCH1 (refs. [7, 8, 10-14]) \\
\hline Head size & Extreme megalencephaly (macrocephaly) \\
Dysmorphic features & $\begin{array}{l}\text { Prominent forehead } \\
\text { Strabismus }\end{array}$ & \\
& Broad nasal bridge & \\
& Frontal hair upsweep & Highly arched eyebrows \\
& Down slanting palpebral & Low-set ears \\
& fissures & Symmetrical post-axial cutaneous appendages (skin \\
& Short nose with broad tip & tags) on both hands \\
& Long philtrum & \\
& Abnormally modelled ears & \\
& & \\
& & \\
Brain imaging & Partial agenesis of the corpus callosum \\
& Enlarged ventricles & \\
Neurological signs & Global developmental delay separated leaves of the septum pellucidum \\
& $\begin{array}{l}\text { Profound intellectual disability } \\
\text { Generalized hypotonia }\end{array}$ \\
\hline
\end{tabular}


can hypothesize that the extreme large volume of the brain tissue in our patient can partly be related to persistent $\mathrm{HH}$ pathway activation [20].

Further studies on possible functional interactions between FOXP1 and PTCH1 may clarify the molecular basis of this phenotype.

Acknowledgements We thank the members of the patient's family for their cooperation.

Funding This study was supported by the GINOP-2.3.2-15-2 grant (to TK and ZM) provided by the National Research, Development and Innovation Office (Hungary).

\section{Compliance with ethical standards}

Ethical approval Written informed parental consent has been obtained. The study was approved by the Human Investigation Review Board at Albert Szent-Györgyi Clinical Centre, University of Szeged, Hungary.

Conflict of interest The authors declare that they have no conflict of interest.

\section{References}

1. Golson ML, Kaestner KH. Fox transcription factors: from development to disease. Development. 2016;143:4558-70.

2. Hamdan FF, Daoud H, Rochefort D, Piton A, Gauthier J, Langlois $\mathrm{M}$, et al. De novo mutations in FOXP1 in cases with intellectual disability, autism, and language impairment. Am J Hum Genet. 2010;87:671-8.

3. Le Fevre AK, Tylor S, Malek NH, Horn D, Carr CW, AbdulRahman OA, et al. FOXP1 mutations cause intellectual disability and a recognizable phenotype. Am $\mathrm{J}$ Med Genet A. 2013;161A:3166-75.

4. Meerschaut I, Rochefort D, Revençu N, Pètre J, Corsello C, Rouleau GA, et al. FOXP1-related intellectual disability syndrome: a recognisable entity. J Med Genet. 2017;54:613-23.

5. Siper PM, De Rubeis S, del Pilar Trelles M, Durkin A, Di Marino D, Muratet F, et al. Prospective investigation of FOXP1 syndrome. Mol Autism. 2017;8:57.

6. Lindström E, Shimokawa T, Toftgård R, Zaphiropoulos PG. PTCH mutations: distribution and analyses. Hum Mutat. 2006;27:215-9.

7. Reinders MG, van Hout AF, Cosgun B, Paulussen AD, Leter EM, Steijlen PM. et al. New mutations and an updated database for the patched-1 (PTCH1) gene. Mol Genet Genomic Med. 2018;6:409_ 15.

8. Fujii K, Miyashita T. Gorlin syndrome (nevoid basal cell carcinoma syndrome): update and literature review. Pediatr Intern. 2014;56:667-74.

9. Mirzaa GM, Poduri A. Megalencephaly and hemimegalencephaly: breakthroughs in molecular etiology. Am J Med Genet C. 2014;166C:156-72.

10. Kimonis VE, Goldstein AM, Pastakia B, Yang ML, Kase R, DiGiovanna JJ, et al. Clinical manifestations in 105 persons with nevoid basal cell carcinoma syndrome. Am J Med Genet. 1997;69:299-308.

11. Kimonis VE, Mehta SG, DiGiovanna JJ, Bale SJ, Pastakia B. Radiological features in 82 patients with nevoid basal cell carcinoma (NBCC or Gorlin) syndrome. Genet Med. 2004;6:495-502.

12. Kimonis VE, Singh KE, Zhong R, Pastakia B, DiGiovanna JJ, Bale SJ. Clinical and radiological features in young individuals with nevoid basal cell carcinoma syndrome. Genet Med. 2013;15:79-83.

13. Veenstra-Knol HE, Scheewe JH, van der Vlist GJ, van Doorn ME, Ausems MGEM. Early recognition of basal cell naevus syndrome. Eur J Pediatr. 2005;164:126-30.

14. Shiohama T, Fujii K, Miyashita T, Mizuochi H, Uchikawa H, Shimojo N. Brain morphology in children with nevoid basal cell carcinoma syndrome. Am J Med Genet. 2017;173:946-52.

15. Sollis E, Graham SA, Vino A, Froehlich H, Vreeburg M, Dimitropoulou $\mathrm{D}$, et al. Identification and functional characterization of de novo FOXP1 variants provides novel insights into the etiology of neurodevelopmental disorder. Hum Mol Genet. 2016;25:54657.

16. Estruch SB, Graham SA, Quevedo M, Vino A, Dekkers DHW, Deriziotis P, et al. Proteomic analysis of FOXP proteins reveals interactions between cortical transcription factors associated with neurodevelopmental disorders. Hum Mol Genet. 2018;27:121227.

17. Braccioli L, Vervoort SJ, Adolfs Y, Heijnen CJ, Basak O, Pasterkamp RJ, et al. FOXP1 promotes embryonic neural stem cell differentiation by repressing Jagged1 expression. Stem Cell Rep. 2017;9:1530-45.

18. Li X, Xiao J, Fröhlich H, Tu X, Li L, Xu Y, et al. Foxp1 regulates cortical radial migration and neuronal morphogenesis in developing cerebral cortex. PLoS ONE. 2015;10:e0127671.

19. Usui N, Araujo DJ, Kulkarni A, Co M, Ellegood J, Harper M, et al. Foxp1 regulation of neonatal vocalizations via cortical development. Genes Dev. 2017;31:2039-55.

20. Briscoe J, Thérond PP. The mechanism of Hedgehog signalling and its roles in development and disease. Nat Rev Mol Cell Biol. 2013;14:416-29. 